\title{
Association of -238G/A and -863C/A polymorphisms in the TNF-a gene with chronic obstructive pulmonary disease based on a meta-analysis
}

\author{
K. Cui, X.Y. Ge and H.L. Ma \\ Department of Epidemiology and Biostatistics, School of Public Health, \\ Liaoning Medical University, Jinzhou Liaoning, China \\ Corresponding author: H.L. Ma \\ E-mail: mahonglinly@163.com
}

Genet. Mol. Res. 12 (4): 4981-4989 (2013)

Received February 25, 20123

Accepted July 4, 2013

Published October 24, 2013

DOI http://dx.doi.org/10.4238/2013.October.24.10

\begin{abstract}
Chronic obstructive pulmonary disease (COPD) is a chronic systemic inflammatory disease; increasing evidence indicates that the TNF- $\alpha$ polymorphism is associated with progression of this disease. Few studies have focused upon association between TNF- $\alpha$ $-238 \mathrm{G} / \mathrm{A}$ or $-863 \mathrm{C} / \mathrm{A}$ polymorphism and COPD risk. Reported associations have been controversial because of small sample size and varied study design among the different studies. We performed a metaanalysis to assess the correlation of these two polymorphisms in the TNF- $\alpha$ gene with COPD risk. A comprehensive search was conducted to identify all published articles on the association between TNF- $\alpha$ $-238 \mathrm{G} / \mathrm{A}$ or $-863 \mathrm{C} / \mathrm{A}$ polymorphism and COPD risk from different databases. Pooled odds ratios (ORs) with 95\% confidence intervals (CI) were calculated, and the heterogeneity and publication bias were assessed. Eight articles with 10 eligible studies met our inclusion criteria; six studies were of the $-238 \mathrm{G} / \mathrm{A}$ polymorphism and the others involved the $-863 \mathrm{C} / \mathrm{A}$ polymorphism. In the case of the $-863 \mathrm{C} / \mathrm{A}$
\end{abstract}


polymorphism, significant association was detected only in Asians in the A allele carriers (GA+AA versus GG genotype) and allele (A versus $\mathrm{G}$ allele) model $(\mathrm{OR}=0.505,95 \% \mathrm{CI}=0.321-0.795$ and $\mathrm{OR}$ $=0.560,95 \% \mathrm{CI}=0.368-0.851$, respectively). However, no significant association was detected for the $-238 \mathrm{G} / \mathrm{A}$ polymorphism. No evidence of between-study heterogeneity and publication bias was detected. We suggest a potentially protective role of the A allele in the TNF- $\alpha-863 \mathrm{C} /$ A polymorphism against developing COPD in Asians. This hypothesis needs further studies for confirmation.

Key words: Chronic obstructive pulmonary disease; Meta-analysis; Tumor necrosis factor-alpha; Polymorphism

\section{INTRODUCTION}

Chronic obstructive pulmonary disease (COPD) is a chronic systemic inflammatory disease and is the major cause of morbidity and mortality all over the world (Yanbaeva et al., 2006; Chen et al., 2010; Zhan et al., 2011). Increasing studies have indicated that tumor necrosis factor-alpha (TNF- $\alpha$ ) plays a key role in the progression of COPD, where elevated TNF- $\alpha$ levels have been detected in sputum, bronchoalveolar lavage fluid, and bronchial biopsies of COPD patients (Keatings et al., 1996; Mueller et al., 1996; Sun et al., 1998). Several single nucleotide polymorphisms, such as $-238 \mathrm{G} / \mathrm{A},-308 \mathrm{G} / \mathrm{A},-376 \mathrm{G} / \mathrm{A},-863 \mathrm{C} / \mathrm{A},+489 \mathrm{G} / \mathrm{A}$, $-857 \mathrm{~T} / \mathrm{C}$, and $-1031 \mathrm{~T} / \mathrm{C}$, have been identified in the TNF- $\alpha$ gene, which is located in the class III region of the major histocompatibility complex on chromosome 6 , the most polymorphic region of the human genome (Brogger et al., 2006; Papatheodorou et al., 2007; Trajkov et al., 2009; Cordoba-Lanus et al., 2011). Among the above-mentioned polymorphisms, -308G/A was the best studied, and the role of increasing COPD risk of the A allele in this polymorphism was identified in Asians (Kuçukaycan et al., 2002; Hegab et al., 2005; Hsieh et al., 2008; Zhan et al., 2011; Zhang et al., 2011). The -238G/A and -863C/A are two other polymorphisms in the promoter region of the TNF- $\alpha$ gene. The former polymorphism is known to increase TNF- $\alpha$ gene transcription in vitro (Bayley et al., 2001), and the latter has also been suggested to influence TNF- $\alpha$ expression, possibly through allele-specific binding to nuclear factor- $\mathrm{\kappa B}$ and its effect on chromatin remodeling (Skoog et al., 2006). Few studies have focused on the correlation of COPD risk with the two polymorphisms. However, the results are still controversial because of the small size and varied study design among different studies. Hence, in the present study, we conducted a meta-analysis to assess the association between both $-238 \mathrm{G} / \mathrm{A}$ and $-863 \mathrm{C} / \mathrm{A}$ polymorphisms in the TNF- $\alpha$ gene and COPD risk.

\section{MATERIAL AND METHODS}

\section{Search strategy}

In the present, we respectively conducted a meta-analysis to assess the correlation of COPD risk with the two TNF- $\alpha$ gene polymorphisms, one for the $-238 \mathrm{G} / \mathrm{A}$ (rs361525) polymorphism and the other for the -863C/A (rs1800630) polymorphism. A search was made for 
potentially relevant articles published in English or Chinese in the following electronic databases: PubMed, ISI Web of Science, China National Knowledge Infrastructure, Database of Chinese Scientific and Technical Periodicals, China biology medical literature database, and Wanfang Data. The search strategy used the following key words: "TNF", "tumor necrosis factor", "polymorphism", "SNP", "COPD", and "chronic obstructive pulmonary disease". In the present study, an upper date limit of December 1, 2012 was applied, with no lower date limit. Additional studies were identified by manually reviewing the bibliographies of relevant articles as well as relevant review articles.

\section{Inclusion criteria}

All identified articles were reviewed by two independent investigators to determine whether a study was eligible for inclusion in the present study. The inclusion criteria were as follows: 1) case-control study published as an original study to assess the association between $-238 \mathrm{G} / \mathrm{A}$ or $-863 \mathrm{C} / \mathrm{A}$ polymorphism in the TNF- $\alpha$ gene and risk of COPD; 2 ) numbers in case and control groups reported for each genotype, or data provided from which numbers could be calculated; 3) case and control groups unrelated and drawn from the same temporally and geographically defined underlying population. A consensus with a third reviewer was needed, if there was disagreement between the two investigators about the eligibility of an article.

\section{Data extraction and quality assessment}

Two independent investigators extracted data from each study, and discrepancies were resolved by consensus of all investigators. The following data were collected from each study: name of the first author, publication year, country/territory, ethnicity of the study population, source of control subjects, mean age, male gender percentage in cases and controls, smoking status, genotyping methods, definition of COPD, matched methods, numbers of cases and controls, and genotype and allele distribution.

The Newcastle-Ottawa quality assessment scale was applied to assess the quality of each study by two investigators. The quality of case-control studies was evaluated in three major components, including selection of cases and controls, comparability of cases and controls, and ascertainment of exposure.

\section{Statistical analysis}

The Fisher exact test was applied to assess deviation from Hardy-Weinberg equilibrium (HWE) for each genotype distribution in the control group. Pooled measures were calculated as the inverse variance-weighted mean of the logarithm of the odds ratio (OR) with $95 \%$ confidence interval (CI). The ORs with $95 \% \mathrm{CI}$ for the A allele carriers (GA+AA versus GG genotype) and allele model (A versus $\mathrm{G}$ allele) of the TNF-238 polymorphism, and that for the A allele carriers $(\mathrm{CA}+\mathrm{AA})$ versus $\mathrm{CC}$ genotype and A allele versus $\mathrm{C}$ allele of the TNF-863 polymorphism were calculated. Heterogeneity among studies was assessed by the $I^{2}$ statistic, which described the proportion of total variation attributable to between-study differences or heterogeneity as opposed to random error or chance. If $I^{2}>50 \%$, the DerSimonian and Laird random-effect model was adopted as the pooling method; otherwise, the fixed-effect model was used. A sensitivity 
analysis was conducted by excluding one study at a time to evaluate how robust the pooled estimator was to removal of individual studies. Publication bias was estimated with the asymmetry linear regression of the Egger test, and displayed as a funnel plot. All statistical analyses were performed with STATA version 9.2 (Stata Corporation, College Station, TX, USA). All reported probabilities were two-tailed, with $\mathrm{P}<0.05$ considered to be statistically significant.

\section{RESULTS}

\section{Study characteristics}

Eight articles (Kucukaycan et al., 2002; Brogger et al., 2006; Papatheodorou et al., 2007; Gingo et al., 2008; Gong et al., 2008; Trajkov et al., 2009; Chen et al., 2010; Bi et al., 2011 ) with ten eligible studies met the inclusion criteria. Among all the included studies, six (Kuçukaycan et al., 2002; Brogger et al., 2006; Papatheodorou et al., 2007; Gingo et al., 2008; Gong et al., 2008; Trajkov et al., 2009) assessed the association of COPD risk with the -238 polymorphism in the TNF- $\alpha$ gene, while the other four (Papatheodorou et al., 2007; Gingo et al., 2008; Chen et al., 2010; Bi et al., 2011) with the -863 polymorphism. Since both of the two polymorphisms were assessed in two articles (Papatheodorou et al., 2007; Gingo et al., 2008), each article was considered to consist of two studies. Among the studies for the -238 polymorphism, one (Gong et al., 2008) was published in Chinese, with the others in English. Among the four studies about the -863 polymorphism, one (Bi et al., 2011) was published in Chinese, and the others were in English. None of the articles applied a matched-design in its study. The genotype distributions of the -238 and -863 polymorphisms were in HWE in the controls of all the studies, except one. Based on the quality assessment scale for case-control studies, two studies scored 5 points, five scored 6 points, and the other three scored 8 points. General characteristics and the allele and genotype distributions of the studies included in the present meta-analysis are shown in Tables 1 and 2.

\section{Quantitative synthesis}

As shown in Figure 1, for the -238G/A polymorphism in the TNF- $\alpha$ gene, no significant heterogeneity was detected for either the A allele carriers or the allele model $\left(I^{2}=32.0\right.$ and $0.0 \%$, respectively), and the fixed-effect model was employed. No significant correlation between the polymorphism and COPD risk was detected in either model, and the pooled ORs were 0.85 $(95 \% \mathrm{CI}=0.59-1.21)$ and $0.97(95 \% \mathrm{CI}=0.69-1.37)$, respectively. Although the ethnicity of the recruited population was the common resource of the between-study heterogeneity, subgroup analysis stratified by ethnicity could not be conducted for the small number of studies, especially in the Asian ethnic subgroup. As shown in Figure 2, for the -863C/A polymorphism, fixed-effect models were applied for the A allele carriers and the allele model $\left(I^{2}=46.1\right.$ and $42.3 \%$, respectively). Significant associations were detected between the polymorphism and COPD risk under the two models, and the overall pooled ORs were $0.73(95 \% \mathrm{CI}=0.56-0.96)$ and $0.76(95 \% \mathrm{CI}=$ 0.60-0.98), respectively. The subgroup analysis stratified by ethnicity for the two models showed a significant correlation of the polymorphism with COPD risk in Asians $(\mathrm{OR}=0.505,95 \% \mathrm{CI}=$ $0.321-0.795$ and $\mathrm{OR}=0.560,95 \% \mathrm{CI}=0.368-0.851$, respectively), but not in Caucasians $(\mathrm{OR}=$ $0.901,95 \% \mathrm{CI}=0.649-1.279$ and $\mathrm{OR}=0.901,95 \% \mathrm{CI}=0.666-1.219$, respectively). 
TNF gene polymorphism and COPD

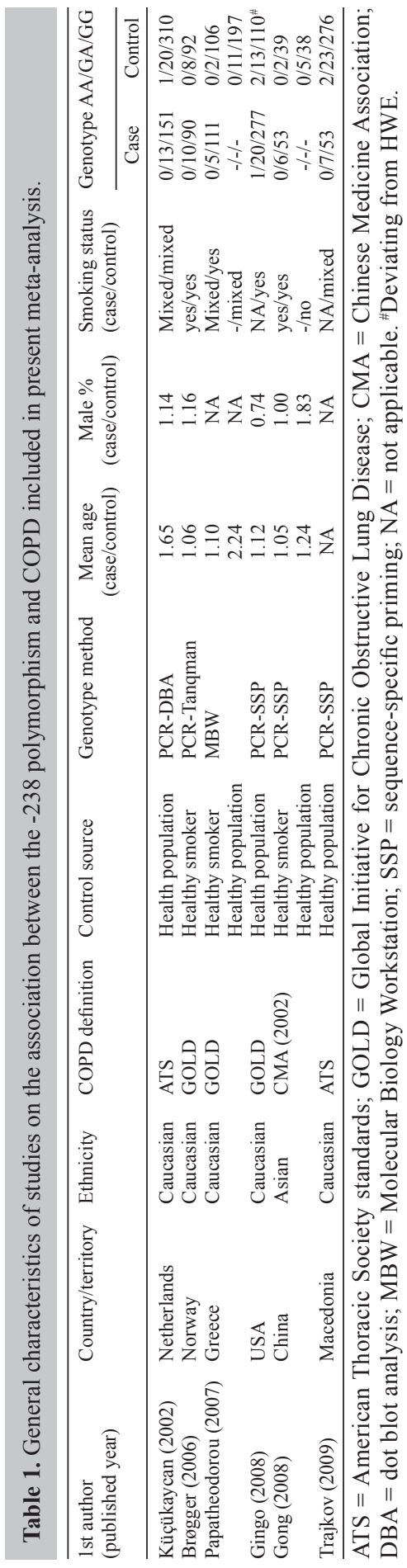


K. Cui et al.

4986

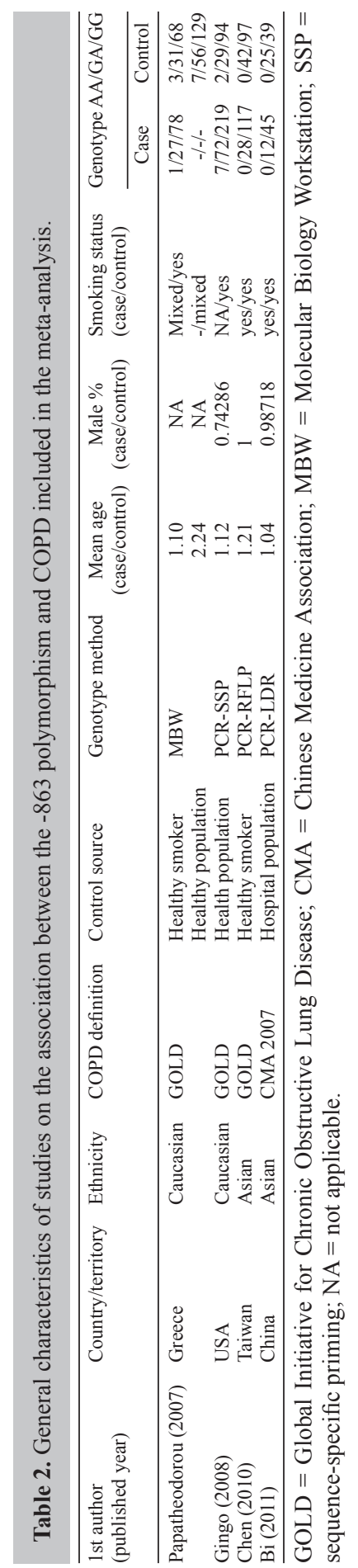



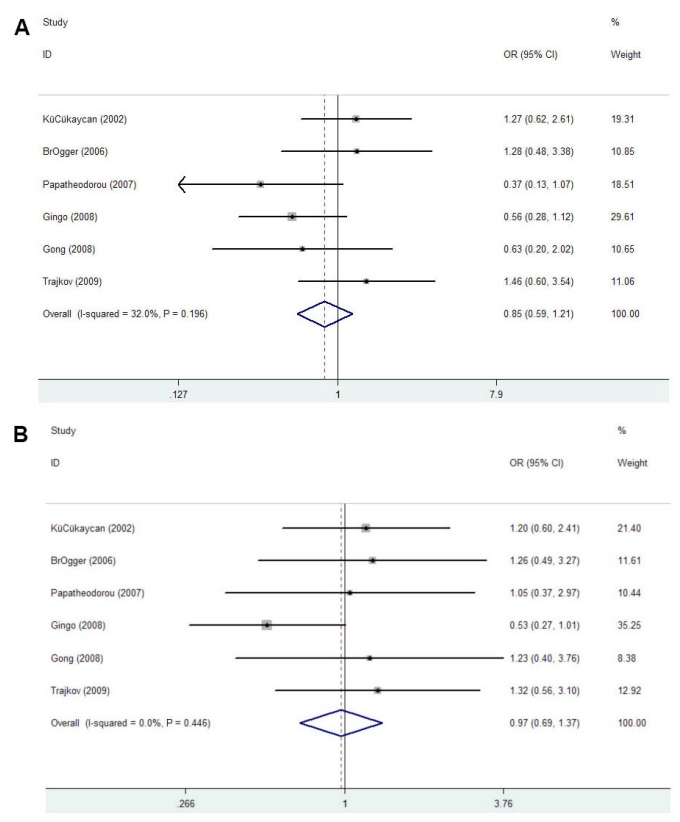

Figure 1. Forest plot of COPD risk associated with the TNF- $\alpha-238 \mathrm{G} / \mathrm{A}$ polymorphism under a fixed-effect model. Each box represents the OR point estimate, and its area is proportional to the weight of the study. The diamond and broken line represent the overall estimate, with $95 \% \mathrm{CI}$ represented by its width. The solid vertical line is set at the null value $(\mathrm{OR}=1.0)$. A. A allele carriers ( $\mathrm{GA}+\mathrm{AA}$ versus $\mathrm{GG}$ genotype). B. Allele model (A versus $\mathrm{G}$ allele).

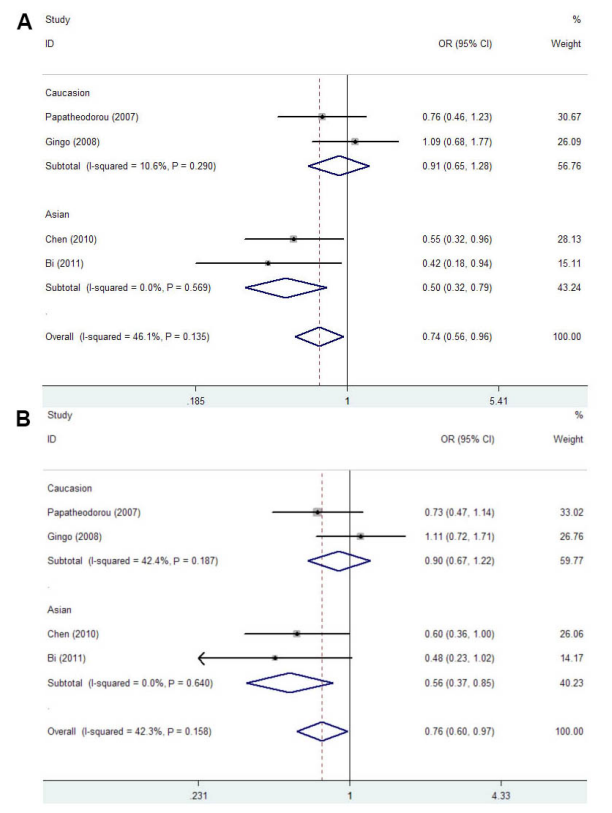

Figure 2. Forest plot of COPD risk associated with the TNF- $\alpha-863 \mathrm{C} / \mathrm{A}$ polymorphism in overall and subgroups, under a fixed-effect model. A. A allele carriers (CA+AA versus CC genotype). B. Allele model (A versus $\mathrm{C}$ allele). 


\section{Sensitivity analysis and publication bias}

After a single study involved in present meta-analysis was excluded at a time, the corresponding results were not materially altered, indicating that the results of the present study were stable. The results of the Egger test and the funnel plot indicated that there was no publication bias in both models of either -238 or -863 polymorphism of the TNF- $\alpha$ gene (data not shown).

\section{DISCUSSION}

Many studies have assessed the association of the polymorphisms in TNF- $\alpha$ gene with COPD risk, and most of these focused on the $-308 \mathrm{G} / \mathrm{A}$ polymorphism, which is located in the promoter region of the gene. Although its association with TNF- $\alpha$ production is still controversial, the role of this polymorphism in increasing COPD risk was identified in two recent metaanalysis studies (Zhan et al., 2011; Zhang et al., 2011). However, the association was detected in Asians but not Caucasians. The $-238 \mathrm{G} / \mathrm{A}$ and $-863 \mathrm{C} / \mathrm{A}$ are two other polymorphisms in the promoter region of the TNF- $\alpha$ gene, and several studies have suggested that these polymorphisms may have an influence on the expression of TNF- $\alpha$. Bayley et al. (2001) suggested that the $-238 \mathrm{G} / \mathrm{A}$ polymorphism could increase the transcription of TNF- $\alpha$ in vitro. Besides, the concentration of TNF- $\alpha$ was found to be 100 -fold higher in airway secretions from the COPD patients with the -238G/A polymorphism than the ones without that in another study (Sapey et al., 2010). Skoog et al. (1999) determined that the -863C/A polymorphism was associated with serum TNF- $\alpha$ concentration and that the carriers of the rare A allele had a lower TNF- $\alpha$ level. However, for the small numbers and varied study design, there was still controversy among the studies focused on the correlation of the two polymorphisms with COPD risk. Therefore, in the present study, we respectively conducted a meta-analysis to assess the association between the polymorphisms and COPD risk.

The results of the present study showed that the -863C/A polymorphism was associated with COPD risk. Since the ethnicity of the recruited population was the common source of the between-study heterogeneity, subgroup analysis stratified by ethnicity was also employed, and the protective role of the $-863 \mathrm{~A}$ allele on COPD risk was identified in Asians but not in Caucasians. However, there was no significant correlation between the $-238 \mathrm{G} / \mathrm{A}$ polymorphism and COPD. Since there was only one study that recruited Asians, we did not conduct a subgroup analysis in the $-238 \mathrm{G} / \mathrm{A}$ polymorphism. Although there was no significant evidence of heterogeneity, publication bias or individual study influence on the pooled effect, the possibilities related to the disease-effect diversity could not be ruled out, because of the small number of studies included in the present meta-analysis.

In conclusion, the present study suggests a potential protective role of the A allele in the TNF- $\alpha-863 \mathrm{C} / \mathrm{A}$ polymorphism against developing COPD in Asians, but no association for the $-238 \mathrm{G} / \mathrm{A}$ polymorphism with COPD risk. Because of the small number of studies included in this article, the results need to be confirmed by further studies.

\section{REFERENCES}

Bayley JP, de Rooij H, van den Elsen PJ, Huizinga TW, et al. (2001). Functional analysis of linker-scan mutants spanning the $-376,-308,-244$, and -238 polymorphic sites of the TNF-alpha promoter. Cytokine 14: 316-323.

Bi ST, Chen S, Ge HB and Li T (2011). Relationship between gene polymorphisms of tumour necrosis factor- $\alpha-863 \mathrm{C} / \mathrm{A}$ and chronic obstructive pulmonary disease. Progr. Mod. Biomed. 11: 4098-4102. 
Brogger J, Steen VM, Eiken HG, Gulsvik A, et al. (2006). Genetic association between COPD and polymorphisms in TNF, ADRB2 and EPHX1. Eur. Respir. J. 27: 682-688.

Chen YC, Liu SF, Chin CH, Wu CC, et al. (2010). Association of tumor necrosis factor-alpha-863C/A gene polymorphism with chronic obstructive pulmonary disease. Lung 188: 339-347.

Cordoba-Lanus E, Baz-Davila R, de-Torres JP, Rodriguez-Perez MC, et al. (2011). TNFA-863 polymorphism is associated with a reduced risk of chronic obstructive pulmonary disease: a replication study. BMC Med. Genet. 12: 132.

Gingo MR, Silveira LJ, Miller YE, Friedlander AL, et al. (2008). Tumour necrosis factor gene polymorphisms are associated with COPD. Eur. Respir. J. 31: 1005-1012.

Gong Y, Jin ML, Ren T and Cai YY (2008). Association of tumor necrosis factor gene promoter polymorphism with chronic obstructive pulmonary disease. Chin. J. Clin. Med. 15: 166-169.

Hegab AE, Sakamoto T, Saitoh W, Nomura A, et al. (2005). Polymorphisms of TNFalpha, IL1beta, and IL1RN genes in chronic obstructive pulmonary disease. Biochem. Biophys. Res. Commun. 329: 1246-1252.

Hsieh MH, Chong IW, Hwang JJ, Lee CH, et al. (2008). Lack of associations between several polymorphisms in cytokine genes and the risk of chronic obstructive pulmonary diseases in Taiwan. Kaohsiung. J. Med. Sci. 24: 126-137.

Keatings VM, Collins PD, Scott DM and Barnes PJ (1996). Differences in interleukin-8 and tumor necrosis factor-alpha in induced sputum from patients with chronic obstructive pulmonary disease or asthma. Am. J. Respir. Crit. Care Med. 153: 530-534.

Kuçukaycan M, Van Krugten M, Pennings HJ, Huizinga TW, et al. (2002). Tumor necrosis factor-alpha +489G/A gene polymorphism is associated with chronic obstructive pulmonary disease. Respir. Res. 3: 29.

Mueller R, Chanez P, Campbell AM, Bousquet J, et al. (1996). Different cytokine patterns in bronchial biopsies in asthma and chronic bronchitis. Respir. Med. 90: 79-85.

Papatheodorou A, Latsi P, Vrettou C, Dimakou A, et al. (2007). Development of a novel microarray methodology for the study of SNPs in the promoter region of the TNF-alpha gene: their association with obstructive pulmonary disease in Greek patients. Clin. Biochem. 40: 843-850.

Sapey E, Wood AM, Ahmad A and Stockley RA (2010). Tumor necrosis factor-\{alpha\} rs361525 polymorphism is associated with increased local production and downstream inflammation in chronic obstructive pulmonary disease. Am. J. Respir. Crit. Care Med. 182: 192-199.

Skoog T, van't Hooft FM, Kallin B, Jovinge S, et al. (1999). A common functional polymorphism (C $\rightarrow$ A substitution at position -863) in the promoter region of the tumour necrosis factor-alpha (TNF-alpha) gene associated with reduced circulating levels of TNF-alpha. Hum. Mol. Genet. 8: 1443-1449.

Skoog T, Hamsten A and Eriksson P (2006). Allele-specific chromatin remodeling of the tumor necrosis factor-alpha promoter. Biochem. Biophys. Res. Commun. 351: 777-783.

Sun G, Stacey MA, Vittori E, Marini M, et al. (1998). Cellular and molecular characteristics of inflammation in chronic bronchitis. Eur. J. Clin. Invest. 28: 364-372.

Trajkov D, Mirkovska-Stojkovikj J, Petlichkovski A, Strezova A, et al. (2009). Association of cytokine gene polymorphisms with chronic obstructive pulmonary disease in Macedonians. Iran. J. Allergy Asthma Immunol. 8: 31-42.

Yanbaeva DG, Dentener MA, Creutzberg EC and Wouters EF (2006). Systemic inflammation in COPD: is genetic susceptibility a key factor? COPD 3: 51-61.

Zhan P, Wang J, Wei SZ, Qian Q, et al. (2011). TNF-308 gene polymorphism is associated with COPD risk among Asians: meta-analysis of data for 6,118 subjects. Mol. Biol. Rep. 38: 219-227.

Zhang S, Wang C, Xi B and Li X (2011). Association between the tumour necrosis factor-alpha-308G/A polymorphism and chronic obstructive pulmonary disease: an update. Respirology 16: 107-115. 